\title{
Management of undifferentiated embryonal sarcoma of the liver in children: A case series and management review
}

\author{
J A Geel, ${ }^{1}$ MB BCh, FCPed, Cert Med Onc (Ped); J A Loveland, ${ }^{2}$ MB BCh, FCS, Cert Ped Surg; G J Pitcher, ${ }^{3}$ MB BCh, FCS; P Beale, ${ }^{2}$ MB BCh FCS, \\ J Kotzen, ${ }^{4}$ MB BCh, FCP; J E Poole, ${ }^{1}$ MB BCh, FCPed

\begin{abstract}
Division of Paediatric Haematology and Oncology, Charlotte Maxeke Academic Hospital, University of the Witwatersrand, Johannesburg, South Africa

Department of Paediatric Surgery, Chris Hani Baragwanath Academic Hospital, University of the Witwatersrand, Johannesburg, South Africa Division of Paediatric Surgery, Department of Surgery, University of Iowa, USA

${ }^{4}$ Department of Radiation Oncology, University of the Witwatersrand, Johannesburg, South Africa
\end{abstract}

Corresponding author: J Loveland (loveland@wol.co.za)

Background. Undifferentiated embryonal sarcoma of the liver (UESL) is a rare neoplasm, and the third-most common paediatric hepatic malignancy. However, no treatment guidelines exist. No randomised, controlled trials support specific combinations of therapy.

Objective. To compare presentation and management of UESL with other series, review the literature, and formulate treatment guidelines. Methods. A retrospective chart review of all hepatic malignancies was conducted from 1996 to 2007 and 5 children with UESL were identified. Management and outcomes were documented. The literature regarding treatment modalities up to September 2012 was reviewed. Results. Over a period of 11 years, 5 patients presented. All underwent surgery and 4 received chemotherapy. One received radiotherapy at relapse. Three are disease-free with follow-up of $58-184$ months. One died after relapse, as did the patient whose family declined chemotherapy.

Conclusion. The improved outcomes are consistent with the international experience and are probably related to combined treatment modalities and advances in supportive care. Pre-operative percutaneous biopsy provides no benefit if the lesion is resectable because it may not prove to be diagnostic, and may cause recurrence in the biopsy tract. If resectable, the recommended treatment is primary excision and adjuvant chemotherapy, with radiotherapy in selected cases. If unresectable, open biopsy is necessary to document histology, and neoadjuvant chemotherapy is given prior to resection. If deemed unresectable, liver transplantation is considered.

S Afr Med J 2013;103(10):728-731. DOI:10.7196/SAMJ.6058

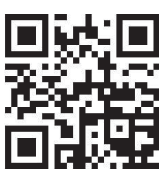

Undifferentiated embryonal sarcoma of the liver (UESL) is a rare hepatic neoplasm that occurs mainly in children. After hepatoblastoma (HB) and hepatocellular carcinoma (HCC), it is the third most common malignant liver tumour in children. ${ }^{[1]}$ Most are diagnosed in the 6 - 10-year age group, with $88 \%$ of patients diagnosed before the age of 15 years. ${ }^{[2]}$ This falls between the peak ages of incidence for HB ( $<2$ years) and HCC ( $>10$ years). Although there are diagnostic criteria on clinical, radiological and histological grounds, ${ }^{[3.5]}$ there is no treatment consensus. ${ }^{[6]}$ Early literature reports a poor prognosis with limited survival, and long-term survival rates are difficult to assess due to relatively short follow-up. Subsequent reports suggest significantly improved prognosis with the use of multimodal therapy, ${ }^{[6-8]}$ which may be secondary to one of many factors. Some units favour a sequence of neo-adjuvant chemotherapy, followed by surgery and adjuvant chemotherapy. Others prefer surgery followed by chemotherapy. Radiation has an uncertain role and the optimal dose has yet to be established.

To determine the optimal course of therapy the 5 cases reported here are compared with documented series, to determine whether there are major differences in presentation, treatment and outcomes, alongside a review of the literature.

\section{Methods}

After institutional ethical approval, case records of all hepatic malignancies treated between July 1996 and December 2007 were retrospectively reviewed. Five patients with histologically proven
UESL presented to the paediatric oncology unit. This state-funded unit provides specialist care to all requiring it, irrespective of their ability to pay. Particular reference was made to mode and duration of presenting symptoms, imaging studies, treatment modalities and outcomes. An English language Pubmed search, limited to paediatric patients, was performed retrospectively from 2012, using the search criteria 'undifferentiated sarcoma of the liver' and 'malignant mesenchymoma'. Publications containing details of surgical procedures and chemotherapy regimens were included, which were composed mainly of retrospective reviews. They were critically assessed to elucidate trends, particularly a relationship between clear surgical margins and improved survival, and the efficacy of particular chemotherapy protocols.

\section{Results}

Five patients -4 females and 1 male, ranging in age from 5 years to 11 years - were diagnosed with UESL. Follow-up ranged in duration from 58 months to 15 years. Two have subsequently died: the first child was operated on but the family elected not to utilise chemotherapy for religious reasons; the second achieved short-term remission but died from recurrence 12 months after diagnosis.

All patients presented with a short duration of abdominal symptoms, including pain, discomfort and distension. All had normal $\alpha$-fetoprotein levels. None had metastatic disease at the time of diagnosis. One patient with an apparently irresectable tumour received neo-adjuvant chemotherapy prior to resection; the remaining 4 underwent primary surgical excision, followed by 
chemotherapy. Of these 4 patients, microscopically clear margins were achieved in 3 . Two had intra-operative spillage of tumour. One suffered spontaneous rupture of the tumour and received brachytherapy when he presented with recurrent disease. All tumours were right-sided, necessitating either right- or extended right hepatectomy. There was no significant post-operative morbidity. Patients presented similarly to other documented cases. There was no unusual delay in presentation, and patients were managed according to available protocols.

\section{Discussion}

Willis first described the pathology of UESL in 1962, classifying it as a rhabdomyoblastic mixed tumour. ${ }^{[0]}$ In 1973 Stanley classified it as a malignant mesenchymoma because of the presence of focal chondroid-appearing cells. ${ }^{[10]}$ Stocker and Ishak definitively described the pathology and adopted the term 'undifferentiated (embryonal) sarcoma of the liver. ${ }^{\text {[3] }}$

At first, imaging studies of UESL may appear discordant: the mass appears solid on ultrasound, but predominantly cystic on CT Scan and magnetic resonance imaging (MRI). The cystic appearance is thought to be due to the high water content of the myxoid stroma. Macroscopic findings correlate more closely with the ultrasound than the CT or MR images. ${ }^{[4,5]}$ UESL are large tumours with haemorrhage, necrosis and pseudocystic areas. Exophytic or pedunculated forms most commonly affect the right lobe of the liver. ${ }^{[3]}$ UESL is composed of atypical spindle, stellate or polygonal cells, which stain positive with periodic acid-Schiff (PAS) stain. There is a clear margin between tumour cells and normal liver, which is often compressed to form a pseudocapsule. In the periphery of the tumour one may find normal hepatocytes and bile ducts, albeit often compressed. Although most cases of UESL are considered to arise de novo, clinical and histological evidence suggests that UESL can arise within mesenchymal hamartomas of the liver (MHL) and similar cytogenetic abnormalities have been shown in both lesions, suggesting a link between the two. ${ }^{[11,12]}$

Many case reports and case series have detailed various combinations of treatment modalities, but only two report patients with UESL prospectively entered into a clinical trial. ${ }^{[1,1,14]}$ Overall survival rates are difficult to assess, as few reports document long term follow-up. Early literature reports a poor prognosis with limited survival. Stocker and Ishak report an $80 \%$ mortality rate in a median period of 11 months. Subsequent articles suggest that the use of multimodal therapy has significantly improved prognosis. ${ }^{[6-8]}$ This improvement in outcome may be related to a modern multidisciplinary approach, including potent combinations of neo- adjuvant and adjuvant chemotherapy, radical surgical resection and radiotherapy, and improved supportive care.

Primary surgical resection remains the diagnostic and therapeutic procedure of choice. However, in patients where UESL is suspected, and resection is deemed unfeasible on imaging, histological confirmation is mandatory. This is because neo-adjuvant protocols differ from those used to treat HB and HCC. Although UESL can be successfully diagnosed using fine-needle aspiration and percutaneous biopsy, ${ }^{[15]}$ there is a theoretically higher risk of peritoneal and tract seeding. ${ }^{[16]}$ Due to the predominantly cystic nature of UESL, percutaneous biopsy also may not provide representative tissue and an open biopsy is preferred. Biopsy is, however, not routine in the work up of HB and HCC, although it is used where there is diagnostic doubt since HB may present similarly to UESL and have low $\alpha$-fetoprotein levels.

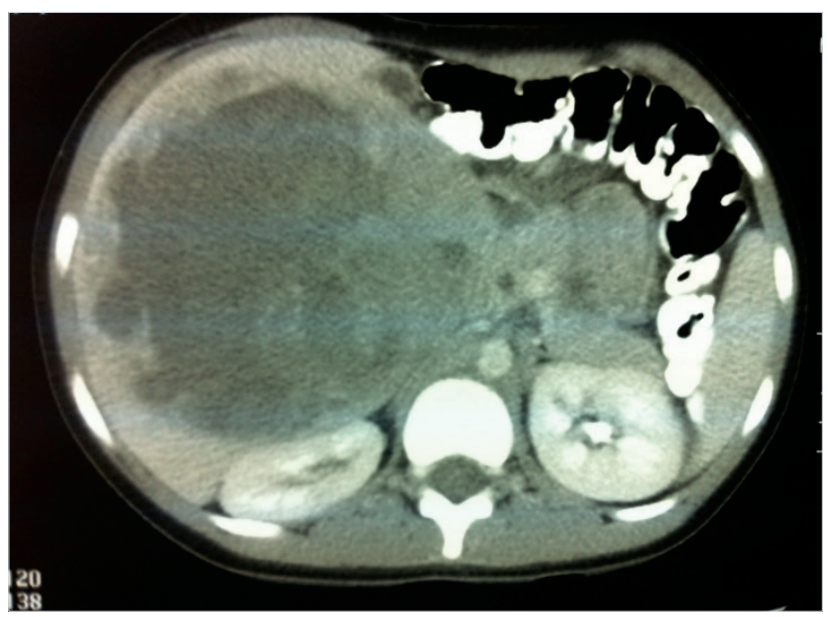

Fig. 1. Typical CT scan of UESL demonstrating mixed solid and cystic lesion.

Table 1. Patient characteristics and outcomes of treatment

\begin{tabular}{|c|c|c|c|c|c|c|c|}
\hline Case & Age & Sex & Chemotherapy & Surgical details & Additional information & Follow up & Status \\
\hline 1 & 8 & $\mathrm{~F}$ & PLADO & $\begin{array}{l}\mathrm{R} \text { hepatectomy } \\
\text { Clear margins }\end{array}$ & & 15 years & NED \\
\hline 2 & 5 & $\mathrm{~F}$ & VIDE & $\begin{array}{l}\mathrm{R} \text { hepatectomy } \\
\text { Clear margins } \\
\text { Spillage }\end{array}$ & & 21 months & LTFU \\
\hline 3 & 6 & $\mathrm{~F}$ & VIDE & $\begin{array}{l}\mathrm{R} \text { hepatectomy } \\
\text { Clear margins } \\
\text { Spillage }\end{array}$ & $\begin{array}{l}\text { Second look surgery: cyst } \\
\text { removed }\end{array}$ & 39 months & NED \\
\hline 4 & 8 & $\mathrm{~F}$ & Declined & $\begin{array}{l}\text { Partial hepatectomy } \\
\text { Excision of part of } \\
\text { diaphragm } \\
\text { Residual disease }\end{array}$ & & 18 months & DOD \\
\hline 5 & 11 & M & VIDE & $\begin{array}{l}\mathrm{R} \text { hepatectomy } \\
\text { spontaneous rupture }\end{array}$ & $\begin{array}{l}\text { Recurrence treated } \\
\text { with brachytherapy radiation }\end{array}$ & 12 months & DOD \\
\hline
\end{tabular}




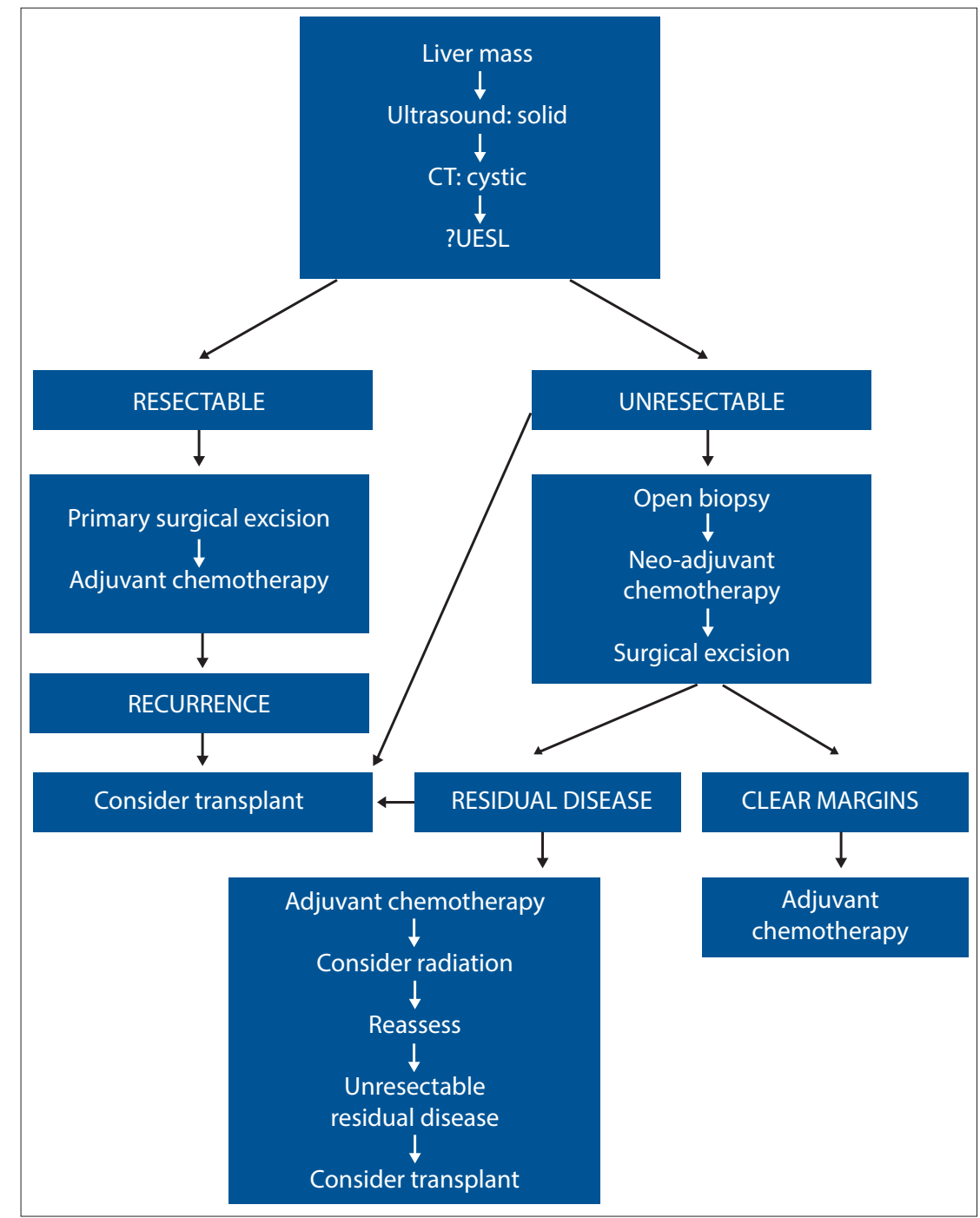

Fig. 2. Proposed treatment algorithm.

Patients with UESL cannot be cured using modalities that exclude surgery. ${ }^{[14]}$ Where the lesion is deemed resectable on available radiology, the ideal approach is primary exploration with a view to complete resection and subsequent adjuvant therapy. Steiner et al. ${ }^{[17]}$ describe second-look laparotomy with biopsy of the tumour bed after initial resection and adjuvant chemotherapy to assess response. Diagnostic radiology improvements render this approach obsolete, and the second-look laparotomy may expose the patient to unnecessary surgery with potential morbidity and mortality.

The sequence of open biopsy, neo-adjuvant chemotherapy, repeat imaging, surgical excision, and adjuvant chemotherapy has markedly reduced tumour size in some cases, with complete resection being achieved in cases that previously could not be adequately surgically excised. ${ }^{[7-20]}$ Liver transplantation has been performed for non- resectable tumours, ${ }^{[2]]}$ or for recurrent disease after volume is reduced, allowing successful resection and consequent improved survival. ${ }^{[19,2,2,27]}$ Postoperative chemotherapy is essential. ${ }^{[8,1,92,26,28,29]}$

Sarcoma and hepatoblastoma chemotherapy protocols appear to demonstrate the greatest efficacy in UESL. Because there were no standard treatment guidelines, the Italian (GCI) and German (CWS) Soft Tissue Sarcoma CooperativeStudyGroupsindependently treated children with UESL according to the childhood rhabdomyosarcoma guidelines. ${ }^{[13]}$ Seventeen patients were prospectively enrolled in studies between 1979 and 1995. They were treated using the same multimodal therapy approach as for patients with sarcomas, namely conservative surgery at diagnosis, multiagent chemotherapy, and second-look operation in cases of residual disease. Of these tumours $62 \%$ showed reduction in volume following chemotherapy. There are more data on the treatment of patients with sarcoma protocols $s^{[7,8,13,14,2,2,2]}$ than those with hepatoblastoma protocols. ${ }^{[17-19,3,0,31]}$ Such reports do not lend themselves to rigorous statistical analysis, but the relatively high survival rate in patients treated on sarcoma protocols implies acceptable efficacy. Since the camptothecin analogues irinotecan and topotecan are promising new agents in treating rhabdomyosarcoma, they may prove to be of use in treating UESL.

An article on UESL from a developing country details the successful treatment of 3 patients with cisplatinum, bleomycin and etoposide, ${ }^{[20]}$ a course of treatment which may have been chosen for its comparatively low cost, despite the incidence of pulmonary fibrosis which limits use of bleomycin in better-resourced centres. All 3 patients were alive at the time of publication, lending support to the use of these drugs in developing countries.

Agents such as paclitaxel, vinorelbine and gemcitabine all offer hope of future treatment options. Oxaliplatin is a newer platinum agent with a more favourable toxicity profile, which is being considered as a possible agent against recurrent hepatoblastoma, and may thus also offer hope in the treatment of UESL.

Stocker and Ishak ${ }^{[3]}$ first reported the use of radiotherapy in the treatment of UESL. Of 31 patients 7 were exposed to radiotherapy, but their outcome is not specifically recorded. However, at a median of 11 months, mortality for all patients was $80 \%$. There has been little subsequent data specific to the use of radiotherapy, as many series do not include it in their protocols. Of those including radiotherapy, its use was often only in isolated cases, rather than as part of a standardised protocol. ${ }^{[3,1,1,1,1,19]}$ 
Neither radiotherapy alone, nor the combination of surgery and radiation without chemotherapy, improves survival in UESL. ${ }^{[28]}$ While radiotherapy is not part of the standard treatment protocol for other hepatic malignancies (because the effective tumour dose exceeds hepatic tolerance), a role for it may evolve in the treatment of UESL as soft-tissue sarcomas are typically radiosensitive. Thus, due to the high incidence of local recurrence, radiotherapy may be justified as an adjunct to surgery and chemotherapy. ${ }^{[14,17,19,27,28,32,33]}$ Neo-adjuvant chemoradiation has also been used to reduce tumour size, leading to total resection. ${ }^{[19]}$ Based on largely anecdotal evidence, radiotherapy is reserved for high-risk patients, including those in whom tumour spillage or rupture has occurred or in whom surgical margins are positive. Protocols tend to be individualised, based on the treating facility's local experience.

While the lowest effective radiation dose has yet to be defined, soft tissue sarcomas require doses greater than 50 Gray (Gy). Doses given with apparent success in UESL range from 20 to $40 \mathrm{~Gy}$. The tolerance of more than $50 \%$ of the liver is limited to $30 \mathrm{~Gy}$ (34). As the liver moves with respiration, image-guided radiotherapy may improve the ability to treat without exceeding hepatic tolerance.

Local recurrence and metastatic disease remain the major impediments to long-term, disease-free survival. ${ }^{[27]}$ Due to the rarity of UESL, there are minimal data on which to judge the effectiveness of newer therapies, including radiotherapy and chemotherapy. Further questions to be explored include the possible role of stem cell transplants, and which agents should be used as second-line treatment in cases of relapse. Liver transplantation is a final treatment option in patients with unresectable tumours. In under-resourced developing countries, this route cannot be recommended as routine, due to organ scarcity and prohibitively high costs.

\section{Conclusion}

Prognosis in UESL has improved dramatically with the aggressive use of multimodal therapy. Should the lesion initially be deemed resectable, primary surgical excision to ensure decreased tumour burden before chemotherapy will achieve optimal results. The recommendation is that an open biopsy should be performed when imaging predicts an incomplete excision, followed by neo-adjuvant chemotherapy, repeat imaging, surgical excision, and adjuvant chemotherapy. The small numbers of patients with this condition preclude randomised controlled trials. Guidelines such as these are offered to clinicians to guide their decisions for treatment of this rare childhood tumour.

\section{References}

1. Pizzo PA, Poplack DG, eds. Principles and Practice of Pediatric Oncology. Philadelphia: JB Lipincott Company, 2011:840.

2. Ishak KG, Goodman ZD, Stocker JT. Tumors of the Liver and Intrahepatic Bile Ducts. Washington DC: Armed Forces Institute of Pathology; Atlas of Tumor Pathology; 3rd series, fascicle 31, 2001:313-320. 3. Stocker JT, Ishak KG. Undifferentiated (embryonal) sarcoma of the liver: Report of 31 cases. $\begin{array}{lll}\text { Cancer 1978;42(1):336-348. [http://dx.doi.org/10.1002/1097-0142(197807)42:1<336:AID- } & \end{array}$ CNCR2820420151>3.0.CO;2-V]

4. Ros PR, Olmsted WW, Dachman AH, et al. Undifferentiated (embryonal) sarcoma of the liver: Radiologic-pathologic correlation. Radiology 1986;160(1):141-145.
5. Buetow PC, Buck JL, Pantongrag-Brown L, et al. Undifferentiated (embryonal) sarcoma of the liver: pathological basis of imaging findings in 28 cases. Radiology 1997; 203(3):779-783.

Upadhyaya M, McKiernan P, Hobin D, et al. Primary hepatic sarcomas in children - a singlecenter experience over 19 years. J Pediatr Surg 2010;45(11):2124-2128. [http://dx.doi.org/10.1016/j. jpedsurg.2010.07.016]

7. Kim DY, Kim KH, Jung SE, et al. Undifferentiated (embryonal) sarcoma of the liver: Combination treatment by surgery and chemotherapy. J Pediatr Surg 2002;37(10):1419-1423. [http://dx.doi. treatment by surgery and $c$
org $/ 10.1053 /$ jpsu.2002.35404]

8. Baron P, Majlessipour F, Bedros AA, et al. Undifferentiated embryonal sarcoma of the liver successfully treated with chemotherapy and liver resection. J Gastrointest Surg 2007;11(1):73-75. [http://dx.doi. org/10.1007/s11605-006-0044-4]

9. Willis RA. The Borderland of Embryology and Pathology. 2nd ed. London: Butterworth, 1962

10. Stanley RJ, Dehner LP, Hesker AE. Primary malignant mesenchymal tumors (mesenchymoma) of the liver in childhood: an angiographic-pathologic study of three cases. Cancer 1973;32(4):973-984. [http://dx.doi.org/10.1002/1097-0142(197310)32:4<973::AID-CNCR2820320432>3.0.CO;2-A]

11. Bove KE, Blough RI, Soukup S. Third report of $\mathrm{t}(19 \mathrm{q})(13.4)$ in mesenchymal hamartoma of liver with comments on link to embryonal sarcoma. Pediatr Dev Pathol 1998;1(5):438-442 [http://dx.doi. org/10.1007/s100249900060]

12. Stringer MD, Alizai NK. Mesenchymal hamartoma of the liver: A systematic review. J Pediatr Surg 2005;40(1):1681-1690. [http://dx.doi.org/10.1016/.jpedsurg.2005.07.052]

13. Bisogno G, Pilz T, Perilongo G, et al. Undifferentiated sarcoma of the liver in childhood: A curable disease. Cancer 2002;94(1):252-257.
distion

14. Horowitz ME, Etcubanas E, Webber BL, et al. Hepatic undifferentiated (embryonal) sarcoma and rhabdomyosarcoma in children. Results of therapy. Cancer 1987;59(3):396-402. [http://dx.doi, org/10.1002/1097-0142(19870201)59:3<396::AID-NCR2820590307>3.0.CO;2-W]

15. Pollono DG, Drut R. Undifferentiated (embryonal) sarcoma of the liver: Fine-needle aspiration cytology and preoperative chemotherapy as an approach to diagnosis and initial treatment. A case report. Diagn Cytopathol 1998;19(2):102-106.

16. Faraj W, Mukherji D, El Majzoub N, et al. Primary undifferentiated sarcoma of the liver mistaken for hydatid disease. World J Surg Oncol 2010;8:58-62. [http://dx.doi.org/10.1186/1477-7819-8-58]

17. Steiner M, Bostrum B, Leonard AS, et al. Undifferentiated (embryonal) sarcoma of the liver: A clinicopathological study of a survivor treated with combined technique therapy. Cancer 1989;64(6):1318-1322. [http://dx.doi.org/10.1002/1097-0142(19890915)64:6<1318::AIDCNCR2820640625>3.0.CO;2-1]

18. Harris MB, Shen S, Weiner MA, et al. Treatment of primary undifferentiated sarcoma of the liver with surgery and chemotherapy. Cancer 1984;54(12):2859-2862. [http://dx.doi.org/10.1002/10970142(19841215)54:12<2859::AID-CNCR2820541208>3.0.CO;2-1]

19. Urban CE, Mache CJ, Schwinger W, et al. Undifferentiated (embryonal) sarcoma of the liver in childhood: Successful combined-modality treatment in four patients. Cancer 1993;72(8):2511-2516.

20. Chowdhary SK, Trehan A, Das A, et al. Undifferentiated embryonal sarcoma in children: Beware of the . Chowdhary SK, Trehan A, Das A, et al. Undifferentiated embryonal sarcoma in children: Beware of the
solitary liver cyst. J Pediatr Surg 2004;39(1):e9-e12. [http://dx.doi.org/10.1016/j.jpedsurg.2003.09.036] 21. Dower NA, Smith. Malignant liver tumors in children. Med Pediatr Oncol 2000;34(2):136-140. [http:// dx.doi.org/10.1002/(SICI) 1096-911X(200002)34:2<136::AID-MPO12>3.0.CO;2-A]

22. Kelly MJ, Martin L, Alonso M, et al. Liver transplant for relapsed undifferentiated embryonal sarcoma in a young child. J Pediatr Surg 2009;44(12):e1-e3. [http://dx.doi.org.10.1016/j.jpedsurg.2009.09.008]

23. Chen LE, Shepherd RW, Nadler ML, et al. Liver transplantation and chemotherapy in children with unresectable primary hepatic malignancies: development of a management algorithm. J Pediatr Gastroenterol Nutr 2006;43(4):487-493. [http://dx.doi.org.10.1097/01.mpg.0000235977.59873.e0]

24. OkajimaH,OhyaY,LeeKJ,etal.Managementofundifferentiated sarcoma of the liver including living donor transplantation as a back up procedure. J Pediatr Surg 2009;44:e33-e38

25. Husted TL, Neff G, Thomas MJ, et al. Liver transplantation for primary or metastatic sarcoma to the liver. Am J Transplant 2006;6:392-397. [http://dx.doi.org.10.1111/j.1600-6143.2005.01179.x]
lof

26. Walker NI, Horn MJ, Strong RW, et al. Undifferentiated (embryonal) sarcoma of the liver: Pathologic Walker NI, Horn MJ, Strong RW, et al. Undifferentiated (embryonal) sarcoma of the liver: $\mathrm{P}$.
findings and long-term survival after complete surgical resection. Cancer 1992;69(1):52-59.

27. Newman KD, Schisgall R, Reaman G, et al. Malignant mesenchymoma of the liver in children. J Pediatr Surg 1989;24(8):781-783. [http://dx.doi.org.10.1016/S0022-3468(89)80536-6]

28. Smithson WA, Telander RL, Carney JA. Mesenchymoma of the liver in childhood: Five-year survival after combined-modality treatment. J Pediatr Surg 1982;17(1):70-72. [http://dx.doi.org.10.1016/ S0022-3468(82)80331-X]

29. Ware R, Friedman HS, Filston HC, et al. Childhood hepatic mesenchymoma: Successful treatment with surgery and multiple-agent chemotherapy. Med Pediatr Oncol 1988;16(1):62-65. [http://dx.doi. org.10.1002/mpo.2950160114]

30. Webber EM, Morrison KB, Pritchard SL, Sorenson PH. Undifferentiated (embryonal) sarcoma of the liver: Results of clinical management in one center. J Pediatr Surg 1999;34(11):1641- 1644. [http:// dx.doi.org.10.1016/S0022-3468(99)90634-6]

31. Uchiyama M, Iwafuchi M, Yagi M, et al. Treatment of ruptured undifferentiated sarcoma of the liver in children: A report of two cases and review of the literature. J Hepatobiliary Pancreat Surg 2001;8(1):8791 [http://dx.doi.org.10.1007/s00534017005]

32. McFadden DW, Kelley DJ, Sigmund DA, et al. Embryonal sarcoma of the liver in an adult treated McFadden DW, Kelley DJ, Sigmund DA, et al. Embryonal sarcoma of the liver in an adult treated
with preoperative chemotherapy, radiation therapy, and hepatic lobectomy. Cancer 1992;69(1):39-44. with preoperative chemotherapy, radiation therapy, and hepatic lobectomy. Cancer 1992;69(1):39-4
[http://dx.doi.org.10.1002/1097-142(19920101)69:1<39::AID-CNCR2820690109>3.0.CO;2-G]

33. Cho HS, Park YN, Lyu CJ, et al. Embryonal sarcoma of the liver: Multiple recurrences and histologic dedifferentiation. Med Pediatr Oncol 1999;32(5):386-388. [http://dx.doi.org.10.1002/(SICI)1096911X(199905)32:5<386::AID-MPO15>3.0.CO;2-N]

34. Dawson LA, Ten Haken RK. Partial volume tolerance of the liver to radiation. Semin Radiat Oncol 2005;15(4):279-283. [http://dx.doi.org.10.1016/j.semradonc.2005.04.005]

Accepted 6 December 2012. 